\title{
PRIDDAT, Birger P., Le concert universel. Die Physiokratie. Eine Transformationsphilosophie des 18. Jahrhunderts
}

Guillaume Garner

\section{OpenEdition}

1 Journals

Édition électronique

URL : http://journals.openedition.org/ifha/1123

DOI : 10.4000/ifha. 1123

ISSN : 2198-8943

Éditeur

IFRA - Institut franco-allemand (sciences historiques et sociales)

Référence électronique

Guillaume Garner, «PRIDDAT, Birger P., Le concert universel. Die Physiokratie. Eine

Transformationsphilosophie des 18. Jahrhunderts », Revue de I'IFHA [En ligne], Date de recension, mis en ligne le 01 janvier 2003, consulté le 22 septembre 2020. URL : http://journals.openedition.org/ifha/ 1123 ; DOI : https://doi.org/10.4000/ifha.1123

Ce document a été généré automatiquement le 22 septembre 2020.

(CIFHA 


\title{
PRIDDAT, Birger P., Le concert universel. Die Physiokratie. Eine Transformationsphilosophie des 18. Jahrhunderts
}

\author{
Guillaume Garner
}

1 Cet ouvrage rassemble sept études que l'économiste B.P. a consacrées à la physiocratie. Cinq sont des rééditions d'articles précédemment publiés, tandis que le chapitre sur " Sismondi comme physiocrate aristotélicien » est inédit, l'étude portant sur la physiocratie allemande (chapitre 5) ayant été publiée dans une version quelque peu modifiée dans un autre recueil que l'auteur avait rédigé sur la pensée économique en Allemagne du XVIIe au XXe s. (Produktive Kraft, sittliche Ordnung und geistige Macht. Denkstile der deutschen Nationalökonomie im 18. und 19. Jahrhundert, Marburg : Metropolis, 1998).

2 Dans son introduction, B.P. s'attache à récuser deux erreurs d'interprétation qui sont selon lui souvent commises à propos de la physiocratie. D'une part, elle est fréquemment interprétée de manière anachronique comme une anticipation des théories libérales associées à la " science économique » actuelle : B.P. rappelle à juste titre que si les physiocrates ont amorcé une approche de l'économie comme un tout autonome, leurs conceptions n'en sont pas moins ancrées dans un contexte sociohistorique précis (celui d'une économie et d'une société essentiellement agraires) et dans un cadre philosophique et métaphysique qui est selon lui très imprégné par l'aristotélisme : la physiocratie apparait ainsi comme « une extension de la distinction aristotélicienne entre l'économie naturelle et l'économie non-naturelle (...), chargée de théorèmes sur la productivité de la nature »(p. 7). D'autre part, B.P. réfute les interprétations faisant peu ou prou des physiocrates des précurseurs des écologistes : il s'agit notamment des travaux de Hans Immler publiés dans les années quatre-vingts, au moment où les questions d'environnement ont joué un rôle croissant dans la vie politique et intellectuelle allemande. 
3 Le premier article ( Production et nature $)$ reprend en les développant ces idées : B.P. y souligne notamment qu'à l'inverse des écologistes, les physiocrates ne se soucient nullement d'une éventuelle destruction des ressources naturelles, mais au contraire qu'ils cherchent à prévenir un recul possible de la fertilité naturelle. Il en résulte une conception de l'organisation de l'espace économique qui insiste sur l'extension des superficies cultivées, et une configuration de l'espace de production agricole donnant la priorité à des exploitations vastes et cohérentes. Il y conteste par ailleurs la réalité d'une théorie de la productivité exclusive de la nature, affirmant que le cultivateur, par son travail, participe à la création de la valeur. La continuité qu'il suggère avec Adam Smith et la théorie de la valeur-travail paraît cependant sujette à caution parce que la théorie de la valeur de ce dernier est très complexe et qu'elle n'élimine pas totalement la sphère naturelle de la création des richesses.

4 Tandis que les chapitres 2 et 3 sont consacrés à une traduction et à un commentaire de la «Table raisonnée des principes de l'économie politique » de Dupont de Nemours (1775), le chapitre 4 (« De l'influence chinoise sur la pensée physiocratique ») étudie le texte de Quesnay sur « le despotisme de la Chine» (1767), dans lequel la Chine apparaît comme un plan de projection des conceptions politiques et économiques de Quesnay. Le chapitre 5 montre en quoi les physiocrates allemands, et notamment Schlettwein, ont repris les conceptions de leurs homologues français en les adaptant à la situation sociale et agricole allemande de la fin du XVIIIe s. Les deux derniers chapitres, consacrés respectivement à Sismondi et à Sombart, sont l'occasion d'étudier l'écho dont ont bénéficié les théories physiocratiques après leur quasi-disparition du débat intellectuel européen. B.P. y voit une réhabilitation de l'agriculture d'inspiration romantique chez le premier, et une critique des excès de l'industrialisation chez le second.

5 Guillaume GARNER 